\title{
Comparison of photoelectrochemical water oxidation activity of a synthetic photocatalyst system with photosystem II
}

\author{
Yi-Hsuan Lai, Masaru Kato, Dirk Mersch and Erwin Reisner*
}

Received 4th April 2014, Accepted 12th June 2014

DOI: $10.1039 / c 4 f d 00059 e$

This discussion describes a direct comparison of photoelectrochemical (PEC) water oxidation activity between a photosystem II (PSII)-functionalised photoanode and a synthetic nanocomposite photoanode. The semi-biological photoanode is composed of PSII from the thermophilic cyanobacterium Thermosynechococcus elongatus on a mesoporous indium tin oxide electrode (mesolTO|PSII). PSII embeds all of the required functionalities for light absorption, charge separation and water oxidation and ITO serves solely as the electron collector. The synthetic photoanode consists of a $\mathrm{TiO}_{2}$ and $\mathrm{NiO}_{x}$ coated nanosheet-structured $\mathrm{WO}_{3}$ electrode (nanowO $3\left|\mathrm{TiO}_{2}\right| \mathrm{NiO}_{x}$ ). The composite structure of the synthetic electrode allows mimicry of the functional key features in PSII: visible light is absorbed by $\mathrm{WO}_{3}, \mathrm{TiO}_{2}$ serves as a protection and charge separation layer and $\mathrm{NiO}_{x}$ serves as the water oxidation electrocatalyst. MesolTO|PSII uses low energy red light, whereas nanowO $\mathrm{WO}_{3}\left|\mathrm{TiO}_{2}\right| \mathrm{NiO}_{x}$ requires high energy photons of blue-end visible and UV regions to oxidise water. The electrodes have a comparable onset potential at approximately $0.6 \mathrm{~V}$ vs. reversible hydrogen electrode (RHE). MesolTO|PSII reaches its saturation photocurrent at $0.84 \mathrm{~V}$ vs. $\mathrm{RHE}$, whereas nanoWO 3 $\mathrm{TiO}_{2} \mid \mathrm{NiO}_{x}$ requires more than $1.34 \mathrm{~V}$ vs. RHE. This suggests that mesolTO|PSII suffers from fewer limitations from charge recombination and slow water oxidation catalysis than the synthetic electrode. MesolTOIPSII displays a higher 'per active' site activity, but is less photostable and displays a much lower photocurrent per geometrical surface area and incident photon to current conversion efficiency (IPCE) than nanowO $3\left|\mathrm{TiO}_{2}\right| \mathrm{NiO}_{x}$.

\section{Introduction}

A possible route for the conversion of energy in sunlight into a storable hydrogenbased fuel is solar water splitting. ${ }^{1}$ Photosynthesis uses sunlight, water and $\mathrm{CO}_{2}$ to generate readily useable carbohydrates. At the heart of this process stands the protein complex PSII, which catalyses efficiently the most thermodynamically 
demanding process in biology: solar light-driven water oxidation. As such, PSII not only sets the benchmark for photo-water oxidation catalysis, but also provides an invaluable inspiration for the design of artificial photosynthetic systems. ${ }^{2}$ Essential to the function of PSII is its fine-tuned ability to combine light absorption, multi-charge separation and efficient water oxidation catalysis. Electron transfer in PSII occurs from the excited primary electron donor (P680*), which can be generated upon red light absorption at $680 \mathrm{~nm}$. The electrons are further transferred to the electron acceptor plastoquinones, $\mathrm{Q}_{\mathrm{A}}$ and $\mathrm{Q}_{\mathrm{B}}$, via a pheophytin (Pheo). The generated holes are captured by a tyrosine $\left(\mathrm{Tyr}_{z}\right)$ and subsequently transferred to the $\mathrm{CaMn}_{4}$ oxygen evolving catalyst (OEC) of PSII to oxidise water (Scheme 1a)., ${ }^{2,3}$

Future solar fuel technologies are likely to rely on PEC cells and many electrodes for photo-water oxidation have recently been reported. ${ }^{1 b, 5}$ Despite the immense interest in mimicking PSII, ${ }^{6}$ a direct comparison of a PSII-immobilised electrode with a purely synthetic, PSII-inspired water oxidation photoanode has not yet been presented.

In this discussion, we describe a side-by-side comparison between a PSIIelectrode and a purely synthetic, PSII-inspired water oxidation photoelectrode recently developed in our laboratory. The semi-biological photoanode is composed of PSII isolated from the thermophilic cyanobacterium Thermosynechococcus elongatus on a mesoporous indium tin oxide electrode (mesoITO|PSII, Fig. 1a). ${ }^{7}$ PSII embeds the required functionalities for photo-water oxidation (Scheme 1a) and direct electron transfer is observable from the plastoquinones in PSII to mesoITO, allowing us to use electrochemical methods to study the photocharacteristics of PSII. ${ }^{7}$

The PSII-inspired synthetic photoanode consists of a $\mathrm{TiO}_{2}$ and $\mathrm{NiO}_{x}$ composite on nanosheet-structured $\mathrm{WO}_{3}$ electrode (nanowO $\mathrm{WO}_{3}\left|\mathrm{TiO}_{2}\right| \mathrm{NiO}_{x}$, Fig. 1b). The composite structure of the synthetic electrode allows for partial mimicry of functional key features in PSII: $\mathrm{WO}_{3}$ serves as the visible light harvester, $\mathrm{TiO}_{2}$ acts as a protection and charge separation layer and $\mathrm{NiO}_{x}$ behaves as a water oxidation electrocatalyst in borate solution (Scheme 1b). ${ }^{4} \mathrm{NiO}_{x}$ was reported to have a similar cubane-like structure to the $\mathrm{CaMn}_{4}$ OEC of PSII. ${ }^{8}$ In addition, this

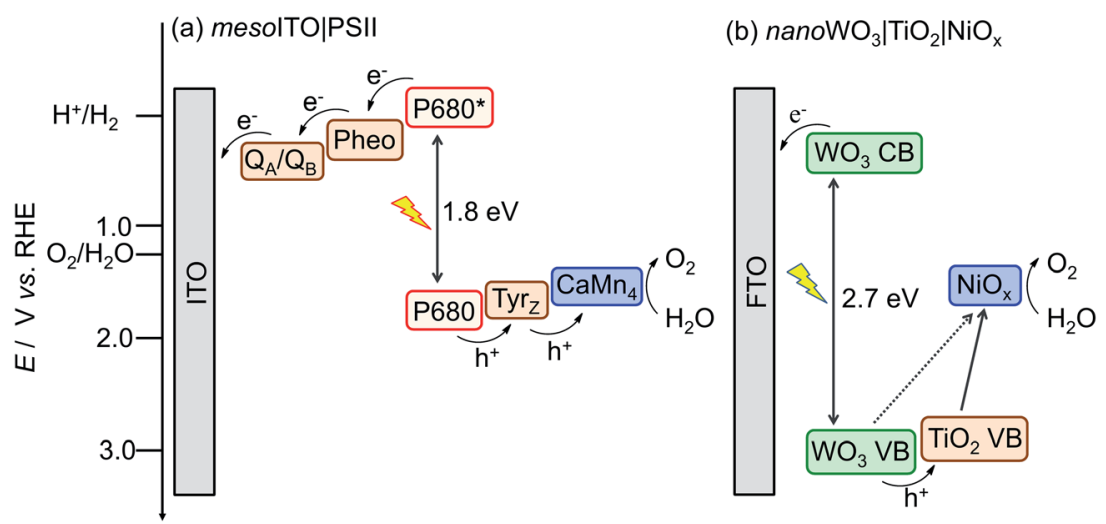

Scheme 1 Energy diagrams for solar light driven water oxidation with (a) mesolTO|PSII and (b) the bio-inspired nanowO $3\left|\mathrm{TiO}_{2}\right| \mathrm{NiO}_{x}{ }^{4}$ 

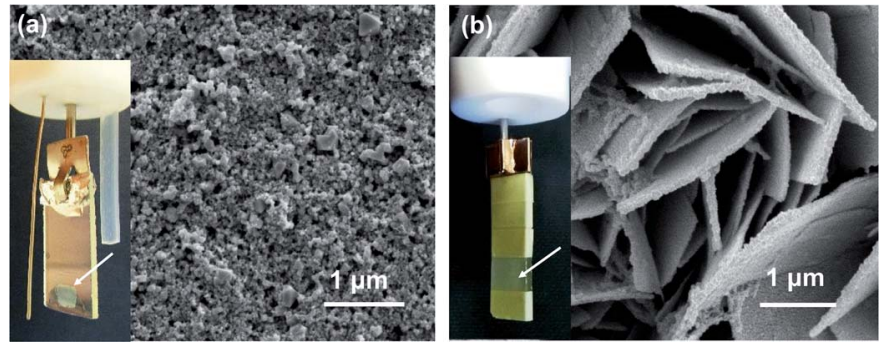

Fig. 1 SEM images of (a) mesolTO and (b) nanoWO $\left|\mathrm{TiO}_{2}\right| \mathrm{NiO}_{x}$. The insets in (a) and (b) show photograph images of the mesolTO|PSII and nanowO $3\left|\mathrm{TiO}_{2}\right| \mathrm{NiO}_{x}$ electrodes, respectively (arrows indicate the exposed working areas).

synthetic electrode is composed of inexpensive materials and is functional under benign conditions (room temperature and $\mathrm{pH} 8$ to 9 aqueous solution). ${ }^{4}$ Functional features of the enzymatic and synthetic systems allow us to compare their performance in $\mathrm{PEC} \mathrm{O}_{2}$-evolution activity, efficiency and stability.

\section{Experimental}

\section{Preparation of mesoITO|PSII electrode}

MesoITO|PSII was prepared according to literature procedures (Scheme 2a). ${ }^{7 a, 9}$ MesoITO was loaded onto an ITO-coated glass slide (VisionTek Systems Ltd) by spreading ITO nanoparticles (Aldrich; $<50 \mathrm{~nm}$ particle size; $27 \mathrm{~m}^{2} \mathrm{~g}^{-1}$ surface area; $90 \% \mathrm{In}_{2} \mathrm{O}_{3}$ and $10 \% \mathrm{SnO}_{2}$ ) with a geometric surface area of $0.25 \mathrm{~cm}^{2}$ (using Scotch ${ }^{\circledR}$ tape $(3 \mathrm{M})$ as spacers to control the active surface area and the film thickness), followed by annealing at $450{ }^{\circ} \mathrm{C}$ for $30 \mathrm{~min} .{ }^{9} \mathrm{PSII}$ dimers were isolated from a thermophilic cyanobacterium, Thermosynechococcus elongatus, and purified following a published protocol. ${ }^{10}$ To assemble mesoITO|PSII, $2 \mu \mathrm{L}$ of a

(a)

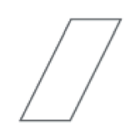

ITO-coated glass

(b)

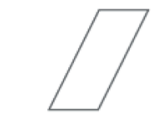

FTO-coated glass

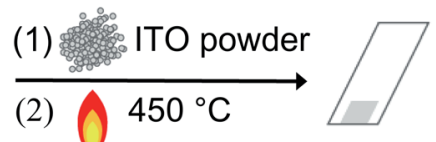

mesolTO

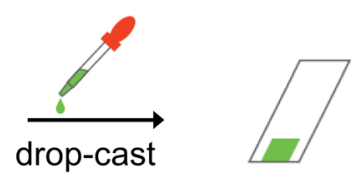

PSII mesolTOIPSII

Scheme 2 Schematic representation of the preparation of (a) mesolTO|PSII and (b) nano $\mathrm{WO}_{3}\left|\mathrm{TiO}_{2}\right| \mathrm{NiO}_{x}$ electrodes. The atoms in TiNi are colour-coded as follows $(\mathrm{H}$ atoms omitted for clarity): Ni (green), Ti (blue), Cl (orange), O (red), C (grey). 
0.54 (mg Chlorophyll a) $\mathrm{mL}^{-1}$ PSII solution was drop-cast onto the mesoITO surface and kept at room temperature in the dark. After $30 \mathrm{~min}$, the PSIImodified mesoITO electrode was used as a working electrode for PEC measurements. ${ }^{7 a, 9}$

\section{Preparation of nanoWO$_{3}\left|\mathrm{TiO}_{2}\right| \mathrm{NiO}_{x}$ electrode}

$\mathrm{NanoWO}_{3}\left|\mathrm{TiO}_{2}\right| \mathrm{NiO}_{x}$ was prepared as described previously (Scheme $2 \mathrm{~b}$ ). ${ }^{4}$ To prepare a $\mathrm{WO}_{3}$ seed layer on a fluoride-doped tin oxide (FTO)-coated glass electrode, a precursor solution containing $\mathrm{H}_{2} \mathrm{WO}_{4}(0.625 \mathrm{~g}$, 99\%; Sigma-Aldrich) and polyvinyl alcohol (0.5 g, 98-99\%, medium molecular weight; Alfa Aesar) in $\mathrm{H}_{2} \mathrm{O}_{2}$ (10 mL, 30\%; Sigma-Aldrich) was spin-coated on the FTO-coated glass (Pilkington;

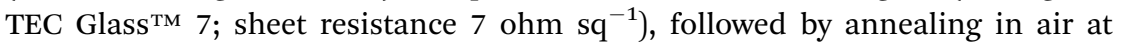
$500{ }^{\circ} \mathrm{C}$ for $2 \mathrm{~h}$. To grow the $\mathrm{WO}_{3}$ nanosheets on the $\mathrm{WO}_{3}$ seed layer-modified FTO-coated glass, a second precursor solution was prepared: a solution of $\mathrm{H}_{2} \mathrm{WO}_{4}$ ( $3 \mathrm{~mL}$ of $0.25 \mathrm{M}$ ) in $\mathrm{H}_{2} \mathrm{O}_{2}$ was added to an aqueous $\mathrm{HCl}$ solution $(3 \mathrm{~mL}, 1 \mathrm{M}$ ) containing oxalic acid (0.2 g, 99\%; Sigma-Aldrich), and then acetonitrile (10 mL, HPLC grade; Fisher Chemicals) was added to this acidic solution. The second precursor solution was put in a $23 \mathrm{~mL}$ Teflon-lined stainless steel autoclave (model 4749, Parr). The $\mathrm{WO}_{3}$ seed layer-modified FTO-substrate was immersed vertically into the second precursor solution in the autoclave, whereupon the autoclave was sealed and heated at $180{ }^{\circ} \mathrm{C}$ for $2.5 \mathrm{~h}$. After growth of the $\mathrm{WO}_{3}$ nanosheets, the resultant electrodes were rinsed with ethanol and then annealed in air for $1 \mathrm{~h}$ at $500{ }^{\circ} \mathrm{C}$.

$\left[\mathrm{Ti}_{2}(\mathrm{OEt})_{9}(\mathrm{NiCl})\right]_{2}$ (TiNi) was synthesised by heating titanium ethoxide $(7.00$ $\mathrm{mL}, 33.4 \mathrm{mmol}$; 99.99\%; Sigma-Aldrich) and nickel(II) chloride (271 mg, 2.09 mmol; >97\%; Sigma-Aldrich) in anhydrous ethanol (7.00 mL, $120 \mathrm{mmol}$ ) in a Teflon-lined autoclave at $150{ }^{\circ} \mathrm{C}$ for $24 \mathrm{~h} .{ }^{11}$ After removal of ethanol, dark yellow crystals of the product were grown from dry toluene at $-14{ }^{\circ} \mathrm{C} . \mathrm{NanoWO}_{3}\left|\mathrm{TiO}_{2}\right| \mathrm{NiO}_{x}$ was assembled by spin coating a fresh solution of TiNi ( $5 \mathrm{mM}$ in toluene) on nano $\mathrm{WO}_{3} . \mathrm{NanoWO}_{3}\left|\mathrm{TiO}_{2}\right| \mathrm{NiO}_{x}$ was dried for at least $30 \mathrm{~min}$ in air at room temperature and then washed with water prior to use as a working electrode.

\section{Physical characterisation}

The surface morphology of mesoITO $\mid \mathrm{PSII}$ and nanoWO $\mathrm{WO}_{3}\left|\mathrm{TiO}_{2}\right| \mathrm{NiO}_{x}$ was characterised with a Philips XL30-SFGE scanning electron microscope (SEM). UV-vis absorption spectra of the electrodes were recorded on a Varian Cary 50 UV-vis spectrophotometer equipped with an energy diffuse reflectance accessory (Barrelino ${ }^{\mathrm{TM}}$ ). The amount of PSII adsorbed on mesoITO was estimated by scraping off the PSII-modified ITO nanoparticles from the ITO substrate, suspending them in $\mathrm{MeOH}$, centrifuging the suspension and analysing the supernatant by UV-vis spectrophotometry. ${ }^{7 a}$ The concentration of Chlorophyll $a(\mathrm{Chl} a)$ in the supernatant was determined using an extinction coefficient equal to 79.95 $(\mathrm{Chl} a \mathrm{mg})^{-1} \mathrm{~mL} \mathrm{~cm}^{-1}$ at $665 \mathrm{~nm}$. The amount of PSII on mesoITO was calculated to be approximately $21.2 \mathrm{pmol} \mathrm{cm} \mathrm{cm}^{-2}$ assuming that the PSII dimer contains $35 \mathrm{Chl}$ $a$ molecules. $^{3 a}$ This PSII loading allowed us to calculate a turnover frequency (TOF) of $0.03\left(\mathrm{~mol} \mathrm{O}_{2}\right)(\mathrm{mol} \mathrm{PSII})^{-1} \mathrm{~s}^{-1}$ for mesoITO|PSII based on photocurrents obtained at $1.23 \mathrm{~V} v$ s. RHE with four electrons per $\mathrm{O}_{2}$ molecule. 


\section{PEC measurements}

PEC measurements were recorded with an Ivium CompactStat potentiostat using a conventional three-electrode system. MesoITO|PSII (exposed projected geometrical area of $0.25 \mathrm{~cm}^{2}$ ) or nanoWO $\mathrm{WO}_{3}\left|\mathrm{TiO}_{2}\right| \mathrm{NiO}_{x}$ (exposed projected geometrical area of $0.5 \mathrm{~cm}^{2}$ ) were used as the working electrodes connected to a Pt foil counter electrode and a $\mathrm{Ag} / \mathrm{AgCl} / \mathrm{KCl}($ sat) reference electrode. All the potentials were converted to the reversible hydrogen electrode (RHE) by using the following equation: $E(\mathrm{~V} v s$. $\mathrm{RHE})=E(\mathrm{~V} v s . \mathrm{Ag} / \mathrm{AgCl})+0.197+0.059 \times \mathrm{pH} .{ }^{12} \mathrm{An}$ aqueous buffered solution ( $\mathrm{pH}$ 6.5) containing $50 \mathrm{mM} \mathrm{KCl}, 15 \mathrm{mM} \mathrm{CaCl}_{2}, 15 \mathrm{mM}$ $\mathrm{MgCl}_{2}$, and $40 \mathrm{mM}$ 2-( $\mathrm{N}$-morpholino)ethanesulfonic acid (MES) was used for the mesoITO|PSII system, whereas the photocurrent responses of nanoWO $3\left|\mathrm{TiO}_{2}\right| \mathrm{NiO}_{x}$ were measured in a potassium borate buffer solution $\left(\mathrm{B}_{\mathrm{i}}, \mathrm{pH}\right.$ 9.2). A solar light simulator (Newport Oriel, Xenon $150 \mathrm{~W}$ ) was used as a light source unless otherwise noted. The light intensity was calibrated to $100 \mathrm{~mW} \mathrm{~cm}^{-2}$ (1 sun). An air mass 1.5 global (AM 1.5G) filter and an IR water filter were used. A $590 \mathrm{~nm}$ cut-off filter (UQG Optics) was used for all PEC experiments with mesoITO|PSII to avoid photoexcitation of ITO. Representative transient photocurrent responses at different potentials of mesoITO|PSII and nanoWO $\mathrm{WiO}_{2} \mid \mathrm{NiO}_{x}$ with two cycles of a dark period followed by irradiation for $20 \mathrm{~s}$ each are shown in Fig. $2 \mathrm{a}$ and b, respectively.

\section{Photon to current conversion efficiency measurements}

A $300 \mathrm{~W}$ Xenon lamp coupled to an MSH300 monochromator (LOT Quantum design) was used for IPCE measurements. The light intensity for the IPCE measurements was measured as a function of wavelength with a photodetector (SEL033/F/QNDS1/W) and power meter (ILT1400). The recorded photocurrents and light intensities at various wavelengths were used to calculate IPCE $(\eta)$ according to the following equation:
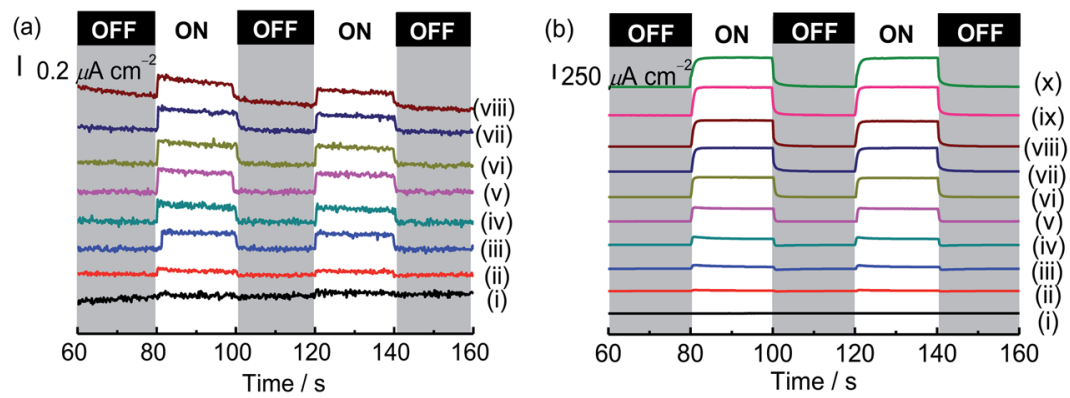

Fig. 2 Representative transient photocurrent response of (a) mesolTO|PSII and (b) nano $\mathrm{WO}_{3}\left|\mathrm{TiO}_{2}\right| \mathrm{NiO}_{x}$ under standardised solar light irradiation (AM 1.5G; ON) and in the dark (OFF) at different applied potentials: (i) 0.54, (ii) 0.64, (iii) 0.74, (iv) 0.84, (v) 0.94, (vi) $1.04 \mathrm{~V}$, (vii), 1.14, (viii), 1.24, (ix) 1.34 and (x) $1.44 \mathrm{~V}$ vs. RHE. Measurements with mesolTO|PSII were recorded in an aqueous $\mathrm{pH} 6.5$ electrolyte solution, whereas nano $\mathrm{WO}_{3}\left|\mathrm{TiO}_{2}\right| \mathrm{NiO}_{x}$ was employed in an aqueous $B_{i}$ buffer (0.1 M, pH 9.2). 


$$
\eta(\%)=\frac{I_{\mathrm{el}}}{I_{\mathrm{ph}}} \times 100=\frac{\frac{J \times 10^{-2}}{F}}{\frac{W \times \lambda \times 10^{-9}}{N_{A} \times h \times c}} \times 100 \approx \frac{1240 \times J}{\lambda \times W}
$$

where $I_{\mathrm{el}}$ is the electron flux at the external circuit $\left(\mathrm{mol} \mathrm{m}^{-2} \mathrm{~s}^{-1}\right), I_{\mathrm{ph}}$ is the incident photon flux $\left(\mathrm{mol} \mathrm{m}^{-2} \mathrm{~s}^{-1}\right), J$ is the measured photocurrent density $\left(\mu \mathrm{A} \mathrm{cm}^{-2}\right)$, $F$ is the Faraday constant ( $96484 \mathrm{~A} \mathrm{~s} \mathrm{~mol}^{-1}$ ), $\lambda$ is the wavelength of light (nm), $W$ is the incident power of the monochromated light $\left(\mathrm{W} \mathrm{m}^{-2}\right), N_{\mathrm{A}}$ is Avogadro's number $\left(6.022 \times 10^{23} \mathrm{~mol}^{-1}\right), h$ is Planck's constant $\left(6.626 \times 10^{-34} \mathrm{~J} \mathrm{~s}\right)$ and $c$ is the speed of light $\left(2.998 \times 10^{8} \mathrm{~m} \mathrm{~s}^{-1}\right)$.

Absorbed photon to current conversion efficiency (APCE) was obtained by dividing the IPCE by the light harvesting efficiency (LHE) at each wavelength: ${ }^{13}$

$$
\begin{gathered}
\operatorname{APCE}(\%)=\operatorname{IPCE}(\%) / \mathrm{LHE} \\
\mathrm{LHE}=1-10^{-A(\lambda)}
\end{gathered}
$$

where $A(\lambda)$ is the absorbance at wavelength $\lambda$.

\section{Results and discussion}

\section{Surface morphologies of mesoITO|PSII and nanoWO ${ }_{3}\left|\mathrm{TiO}_{2}\right| \mathrm{NiO}_{x}$}

MesoITO has a pore diameter of up to $100 \mathrm{~nm}$ (Fig. 1a) and therefore provides a high surface area to integrate PSII (approximately $20.5 \times 10.5 \times 11.0 \mathrm{~nm}^{3}$ for the PSII dimer). ${ }^{3 a}$ MesoITO|PSII was assembled by drop-casting the PSII solution on the mesoITO electrode surface (geometric active surface area: $0.25 \mathrm{~cm}^{2}$, inset of Fig. 1a).

NanoWO $\mathrm{W}_{3}\left|\mathrm{TiO}_{2}\right| \mathrm{NiO}_{x}$ was studied as a PSII-inspired synthetic photoanode. This synthetic photoanode consists of green $\mathrm{WO}_{3}$ nanosheets coated with a uniform composite film containing $\mathrm{TiO}_{2}$ and $\mathrm{NiO}_{x}$ nanoparticles (Fig. 1b). $\mathrm{WO}_{3}$ is an n-type semiconductor with a suitable band gap (approximately $2.7 \mathrm{eV}$ ) and is capable of photo-oxidising water with blue-end visible light (valence band potential at approximately $3 \mathrm{~V}$ vs. RHE, Scheme $1 \mathrm{~b}$ ). The nanosheet structure of $\mathrm{WO}_{3}$ gives a high surface area for light absorption, electrocatalyst loading and a decreased hole diffusion length in the photoanode.

A uniform decoration with $\mathrm{Ti}$ and Ni containing nanoparticles on $\mathrm{WO}_{3}$ can be obtained by spin-coating the molecular TiNi precursor solution onto nanoWO and subsequently hydrolysing the metal-oxide precursor. ${ }^{4}$ TiNi thereby serves as a single source precursor for the formation of $\mathrm{TiO}_{2}$ and $\mathrm{NiO}_{x}$ on nanoWO . The $\mathrm{TiO}_{2}$ layer protects $\mathrm{WO}_{3}$ from the alkaline electrolyte solution and supports charge separation by receiving holes from the photoexcited nanoWO $\mathrm{W}_{3}$. The $\mathrm{NiO}_{x}$ is assembled in situ by photo-generated holes in borate buffer $\left(\mathrm{B}_{\mathrm{i}}\right)$ and promotes water oxidation catalysis. We note that at least some $\mathrm{NiO}_{x}$ is likely to be in close contact with $\mathrm{WO}_{3}$ and direct hole transfer from $\mathrm{WO}_{3}$ to $\mathrm{NiO}_{x}$ is therefore also possible (Scheme 1b).

\section{PEC characteristics}

Fig. 3a shows a schematic $J-V$ curve of an efficient (red dotted line) and inefficient photoanode (black solid line). First, we will discuss (i) the onset potential, (ii) the 
(a)

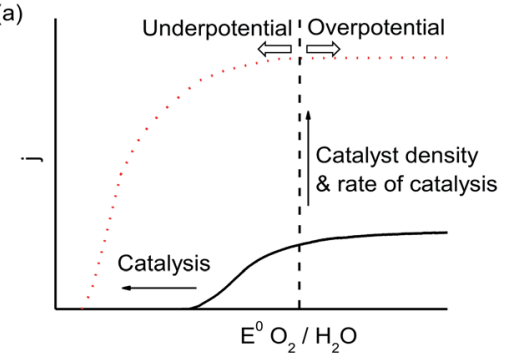

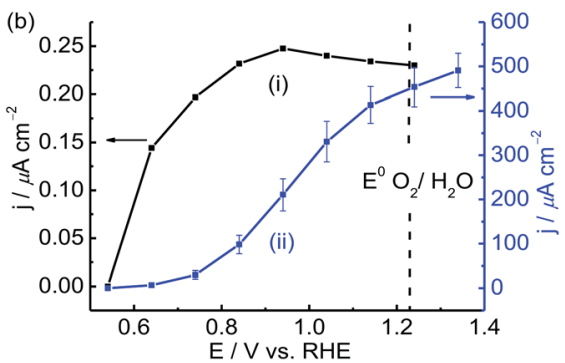

Fig. 3 (a) Performance of an idealised photoanode compared with a photoanode deviated from its ideal case. The solid arrows indicate the parameters that need to be addressed to improve the photoanode. (b) Photocurrent responses at various potentials of a (i) mesoITO|PSII electrode and (ii) nanoWO $3\left|\mathrm{TiO}_{2}\right| \mathrm{NiO}_{x}$ under standardised solar light irradiation (AM 1.5G, $100 \mathrm{~mW} \mathrm{~cm}{ }^{-2}$ ). ${ }^{2,4}$ A $590 \mathrm{~nm}$ cut-off filter was used for mesolTO|PSII. Measurements with electrode (i) were recorded in an aqueous pH $6.5 \mathrm{MES}$ buffer solution and electrode (ii) were recorded in an aqueous $B_{i}$ buffer $(0.1 \mathrm{M}, \mathrm{pH}$ 9.2). The standard deviation for measurements with the mesolTO|PSII electrode (i) was approximately $20 \%$. $E(\mathrm{~V}$ vs. $\mathrm{NHE})=E(\mathrm{~V}$ vs. $\mathrm{RHE})-0.059 \times \mathrm{pH}^{12}$

potential required for reaching plateau current ('saturation photocurrent') and (iii) the plateau current as parameters to characterise the photoelectrodes and compare it to an ideal photoanode. An optimal semiconductor photoanode should have an onset potential close to the conduction band position of $\mathrm{WO}_{3}$ during illumination, whereas we would expect an ideal PSII-based electrode to display photocurrents at slightly more positive potentials than the $\mathrm{Q}_{\mathrm{B}}$ redox potential. At the same time the saturation photocurrent should readily be reached well within the "underpotential" region (at more negative potential than the thermodynamic water oxidation).

In practice however, the onset and saturation potential are shifted to considerably more anodic potentials due to slow catalysis and charge recombination events at the photoanode. The PEC responses of mesoITO|PSII and nano $\mathrm{WO}_{3}\left|\mathrm{TiO}_{2}\right| \mathrm{NiO}_{x}$ were recorded at different potentials under standardised solar light irradiation. A $590 \mathrm{~nm}$ long-pass filter was used for mesoITO|PSII to prevent photoexcitation of ITO. The mean values of the net photocurrent density (J) at specific potentials were obtained by subtracting peak values of the photocurrent density from the background.

The redox potential of plastoquinone $\mathrm{Q}_{\mathrm{A}}$ and $\mathrm{Q}_{\mathrm{B}}$ is approximately $0.3 \mathrm{~V} v$ s. RHE $\left(\sim-0.1 \mathrm{~V} v s\right.$. NHE in $\mathrm{pH}$ neutral environment).${ }^{14}$ MesoITO|PSII was studied in a pH 6.5 MES buffer solution (in the absence of soluble redox mediator) and has an onset potential of $0.60 \mathrm{~V} v s$. RHE during irradiation, which is approximately $0.3 \mathrm{~V}$ more anodic than its theoretical onset potential. We note that direct electron transfer is required from the plastoquinones to ITO for this semi-biological electrode. Coupling of the plastoquinones to the ITO surface is likely to be nonideal, which would explain the anodic shift of the onset potential. The saturation photocurrent of approximately $0.25 \mu \mathrm{A} \mathrm{cm} \mathrm{cm}^{-2}$ was reached close to the onset potential at $0.84 \mathrm{~V} v s$. RHE (trace i, Fig. $3 \mathrm{~b}$ ). This small difference between the onset potential and the saturation photocurrent potential may be explained by the efficient charge separation and facile kinetics for water oxidation in PSII (Scheme 1a). 
Table 1 Comparison of PEC water oxidation activity of mesolTO|PSII and nanoWO $\mathrm{W}_{3}\left|\mathrm{TiO}_{2}\right|$ $\mathrm{NiO}_{x}$

Onset potential, $E_{\mathrm{O}} \Delta E_{\mathrm{O}-\mathrm{P}}{ }^{b} J_{\max }{ }^{c} \quad \mathrm{TOF}^{d} \quad$ Stability, $\mathrm{IPCE}_{\max }^{f} \mathrm{APCE}_{\max }{ }^{f}$ System $^{a}$ (V vs. RHE) (V) $t_{1 / 2}^{e}(\mathrm{~min})(\%)$ (\%)

mesoITO|PSII $\quad 0.60$
nano $\mathrm{WO}_{3}\left|\mathrm{TiO}_{2}\right| \mathrm{NiO}_{x} 0.64$

$\begin{array}{lc}0.2 & 0.3 \\ 0.7 & 500\end{array}$

$\begin{array}{llll}0.03 & \sim 4 & 0.125 & 0.70 \\ 8 \times 10^{-4} & \sim 240 & 50 & 80\end{array}$

${ }^{a}$ MesoITO|PSII was employed as working electrode in a pH 6.5 MES solution. NanoWO $\mathrm{WO}_{3}\left|\mathrm{TiO}_{2}\right| \mathrm{NiO}_{x}$ was studied in an aqueous $\mathrm{pH} 9.2 \mathrm{~B}_{\mathrm{i}}$ buffer $(0.1 \mathrm{M})$. Both electrodes were studied at room temperature with a $\mathrm{Pt}$ counter and a $\mathrm{Ag} / \mathrm{AgCl} / \mathrm{KCl}(\mathrm{sat})$ reference electrode. ${ }^{b}$ Potential difference between onset potential $\left(E_{\mathrm{O}}\right)$ and the potential required to reach the plateau photocurrent $\left(E_{\mathrm{P}}\right) .{ }^{c}$ Saturation photocurrent. ${ }^{d}$ TOF $\left[\mathrm{in} \mathrm{mol} \mathrm{O}_{2}\right.$ (mol catalyst $)^{-1} \mathrm{~s}^{-1}$ ] calculated based on photocurrents obtained at $1.23 \mathrm{~V} v$ s. RHE. For PSII, $100 \%$ Faradaic efficiency was assumed. For $\mathrm{NiO}_{x}$, the TOF was calculated based on evolved $\mathrm{O}_{2}$ gas. ${ }^{4}{ }^{e}$ Calculated at an applied potential of $0.94 \mathrm{~V} v$ s. RHE under solar light irradiation. ${ }_{f}$ Recorded at an applied potential of $0.94 \mathrm{~V}$ vs. RHE $(680 \mathrm{~nm}$ for mesoITO|PSII and $375 \mathrm{~nm}$ for $n a n o \mathrm{WO}_{3}\left|\mathrm{TiO}_{2}\right| \mathrm{NiO}_{x}$ ).

The conduction band of $\mathrm{WO}_{3}$ is situated at approximately $0.3 \mathrm{~V} v$ s. RHE, ${ }^{15}$ which is similar to the redox potential of $\mathrm{Q}_{\mathrm{A}}$ and $\mathrm{Q}_{\mathrm{B}}$ in PSII. Without modification of the surface with $\mathrm{TiO}_{2}$ and $\mathrm{NiO}_{x}$, the onset potential of $n a n o \mathrm{WO}_{3}$ is $0.74 \mathrm{~V} v$ s. RHE, which is notably larger than the onset potential of mesoITO|PSII due to poor catalysis. ${ }^{4}$ Interfacing $\mathrm{TiO}_{2}$ and $\mathrm{NiO}_{x}$ on nanoWO${ }_{3}$ reduces the onset potential to $0.64 \mathrm{~V} v$ s. RHE, a potential which is comparable with mesoITO|PSII. A significant difference in the bias potential required to reach the plateau photocurrent is observed between the two electrode systems. A saturation photocurrent of $500 \mu \mathrm{A}$ $\mathrm{cm}^{-2}$ is reached at $1.34 \mathrm{~V} v$ s. RHE with nano $\mathrm{WO}_{3}\left|\mathrm{TiO}_{2}\right| \mathrm{NiO}_{x}$ compared to $0.84 \mathrm{~V} v$ s. RHE for mesoITO|PSII (Fig. 3b). $\mathrm{TiO}_{2}$ and $\mathrm{NiO}_{x}$ offer only a partial offset for the poor charge recombination kinetics and water oxidation catalytic activity of nano $\mathrm{WO}_{3}$. The plateau photocurrent density is dominated by the amount of the photogenerated holes reaching the surface and oxidising water. Enhancing the catalyst density on the electrode (the amount of photocatalyst based on the geometrical surface area), using a more efficient catalyst or reducing charge recombination within the photoanode increases the photocurrent. ${ }^{1 b, 5,13,16}$ MesoITO|PSII therefore displays more efficient catalysis and suffers from less charge recombination below $1.23 \mathrm{~V}$ vs. RHE (the underpotential region relative to $\left.E^{\circ}\left(\mathrm{H}_{2} / \mathrm{H}_{2} \mathrm{O}\right)\right)$. PSII has an evolutionarily well-developed machinery for the photooxidation of water and immobilised PSII retains these features and behaves more like an ideal photoanode compared to the synthetic electrode. The amount of immobilised PSII on mesoITO is only $21.2 \mathrm{pmol} \mathrm{cm}^{-2}$ due to the large geometrical footprint of the enzyme photocatalyst, whereas $0.78 \mu \mathrm{mol} \mathrm{cm} \mathrm{cm}^{-2}$ of $\mathrm{NiO}_{x}$ is deposited on $n a n o \mathrm{WO}_{3}$ (Table 1). Taking into account the photocurrent density, a much higher single-site catalytic activity was observed for the OEC in PSII with a turnover frequency (TOF) of approximately $0.03 \mathrm{~s}^{-1}$ at $1.23 \mathrm{~V}$ compared to $8 \times$ $10^{-4} \mathrm{~s}^{-1}$ at $1.23 \mathrm{~V} v$ s. RHE for $\mathrm{NiO}_{x}$ in the synthetic system (Table 1$) .{ }^{4}$ On the other hand, the low PSII loading dramatically limits the photocurrent density with mesoITO|PSII and a three-order of magnitude higher photocurrent density per geometric surface area is observed with nanoWO $\mathrm{WO}_{3}\left|\mathrm{TiO}_{2}\right| \mathrm{NiO}_{x}$. 
The electronic absorption spectra in Fig. 4 reveal another key difference between the electrodes. MesoITO|PSII generates $\mathrm{O}_{2}$ during red light irradiation, ${ }^{10 b, 17}$ whereas $\mathrm{WO}_{3}$ is only capable of absorbing photons in the blue and UV regions of the solar spectrum. The synthetic electrode therefore requires substantially more energetic photons to oxidise water. The IPCE spectra show a good agreement with the respective absorption spectra for both electrode systems. IPCE is the measure of the ratio of the photocurrent versus the rate of incident photons as a function of wavelength (eqn (1)). IPCE thereby confirms that the photocurrent of mesoITO|PSII is the result of red light excitation (Fig. 4a), whereas the photocurrent of $n a n o \mathrm{WO}_{3}\left|\mathrm{TiO}_{2}\right| \mathrm{NiO}_{x}$ is generated by blue and UV light (Fig. 4b).

An optimum IPCE of PSII on mesoITO of $0.125 \%$ was obtained at $680 \mathrm{~nm}$, corresponding to the estimated excited-state energy of P680 $\left(1.825 \mathrm{eV}, E_{680}\right)$. For any photon absorbed by PSII, the energy driving the photo-water oxidation is equal to $E_{680}$. The APCE can be readily obtained from the IPCE and the LHE by using eqn (2). LHE quantifies the absorbance of monochromatic light by the electrodes as a function of absorption coefficient (eqn (3)). The LHE of PSII on mesoITO at $680 \mathrm{~nm}$ is approximately $17 \%$, and the corresponding APCE is $0.70 \%$ (Fig. 4 and Table 1). The low quantum efficiency of mesoITO|PSII presumably stems from the low loading of PSII and the random orientation of PSII on the mesoITO electrode, hindering the injection of electrons from PSII to the mesoITO. ${ }^{7}$ However, the APCE of mesoITO|PSII is comparable with the APCE of PSII on a Au substrate. ${ }^{18}$ In contrast, nano $\mathrm{WO}_{3}\left|\mathrm{TiO}_{2}\right| \mathrm{NiO}_{x}$ can only use high-energy photons provided by solar light to promote electron-hole pair separation. NanoWO $\mathrm{WiO}_{3}\left|\mathrm{TiO}_{2}\right| \mathrm{NiO}_{x}$ has a maximum IPCE and APCE of $50 \%$ and $80 \%$ at $375 \mathrm{~nm}$ at an applied potential of $0.94 \mathrm{~V} v$ s. RHE, respectively. The IPCE drops to zero at wavelengths longer than $465 \mathrm{~nm}$, which is consistent with the band gap of $\mathrm{WO}_{3}\left(2.7 \mathrm{eV}, E_{\mathrm{WO} 3}\right)$. Thus, more light energy input $\left(E_{\mathrm{WO} 3}-E_{680}=0.9 \mathrm{eV}\right)$ is required to photo-oxidise water and to promote electrons at approximately $0.64 \mathrm{~V} v s$. RHE with the synthetic hybrid electrode. The nano $\mathrm{WO}_{3}\left|\mathrm{TiO}_{2}\right| \mathrm{NiO}_{x}$ electrode requires an additional energy input to compensate for less efficient water oxidation by $\mathrm{NiO}_{x}$ compared to $\mathrm{CaMn}_{4}$ OEC in PSII.
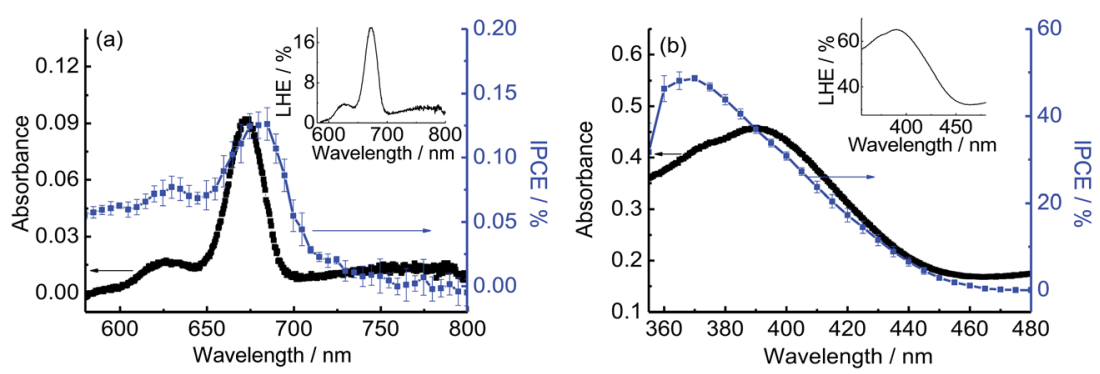

Fig. 4 Diffuse reflectance UV-vis absorption and IPCE spectra of (a) mesolTO|PSII and (b) nanoWO $3\left|\mathrm{TiO}_{2}\right| \mathrm{NiO}_{x}$. IPCE spectra were recorded at an applied potential of $0.94 \mathrm{~V} v \mathrm{~V}$. RHE. Measurements were recorded in an aqueous $\mathrm{pH} 6.5 \mathrm{MES}$ buffer with mesolTOIPSII and in an aqueous $\mathrm{pH} 9.2 \mathrm{~B}_{\mathrm{i}}$ buffer with nanowO $\mathrm{WH}_{3}\left|\mathrm{TiO}_{2}\right| \mathrm{NiO}_{x}$. Insets show the corresponding LHE. 


\section{Photostability}

From a practical standpoint, the long-term photostability of the electrodes in aqueous solution is an important criterion. MesoITO|PSII has a half-life time of approximately $4 \mathrm{~min}$ and complete loss of activity is observed within $30 \mathrm{~min}$ at an applied potential of $0.94 \mathrm{~V}$ vs. RHE (Fig. $5 \mathrm{a}$ and Table 1). The short lifetime of mesoITO|PSII can be attributed to the intrinsic photo-instability of PSII in the presence of intense light irradiation (with no repair machinery being available in isolated PSII) as well as the fragility of the PSII-ITO interface (film loss of PSII). Under strong light irradiation, over-accumulation of excited states leads to excessive charge generation in PSII and results in the generation of Chl $a$ triplet states. These react with triplet oxygen to produce singlet oxygen, which is understood to be one of the reasons for the enhanced damage of PSII. ${ }^{19}$

The synthetic nano $\mathrm{WO}_{3}\left|\mathrm{TiO}_{2}\right| \mathrm{NiO}_{x}$ displays much greater photostability. A half-life time of approximately four hours was observed during solar light irradiation, and a complete loss of activity was only observed after $24 \mathrm{~h}$ (Fig. $5 \mathrm{~b}$ and Table 1). Bare $\mathrm{WO}_{3}$ is known to be soluble in alkaline solution and easily damaged by side reactions during photo- $\mathrm{O}_{2}$ evolution., ${ }^{4,20}$ However, the $\mathrm{TiO}_{2}$ and $\mathrm{NiO}_{x}$ coatings stabilise $\mathrm{WO}_{3}$ by acting as a protective layer and an electrocatalyst, respectively.

\section{Comparison of the semi-biological and synthetic electrode}

MesoITO|PSII shows more efficient 'single-site' catalysis and superior resistance to charge recombination than nanoWO $\mathrm{WO}_{3}\left|\mathrm{TiO}_{2}\right| \mathrm{NiO}_{x}$. This is evident from the higher TOF of PSII and a $0.5 \mathrm{~V}$ more cathodic potential to reach the photocurrent plateau for mesoITO|PSII (Table 1). In this respect, the PEC response of mesoITO|PSII shown in Fig. 3 is closer to an "ideal" photoanode. Moreover, PSII is capable of photo-oxidising water with low energy "red photons", whereas $\mathrm{WO}_{3}$ requires higher energy photons to compensate for significantly higher charge recombination and less efficient $\mathrm{NiO}_{x}$ OEC. Interfacing nanoWO $3\left|\mathrm{TiO}_{2}\right| \mathrm{NiO}_{x}$ with another
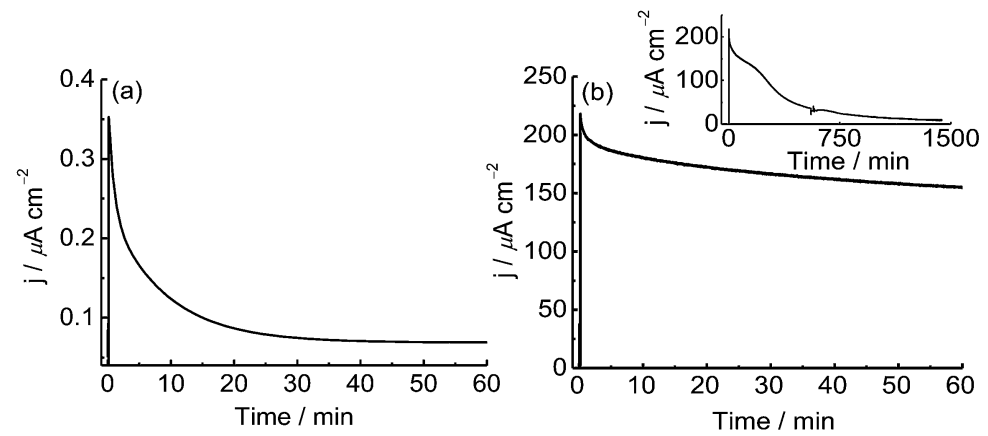

Fig. 5 Chronoamperometric measurements at $0.94 \mathrm{~V}$ vs. RHE. Photocurrent profiles of (a) mesolTO|PSII and (b) nanoWO $\mathrm{WiO}_{2} \mid \mathrm{NiO}_{x}$ under standardised solar light irradiation (AM $1.5 \mathrm{G}, 100 \mathrm{~mW} \mathrm{~cm}^{-2}$ ) are shown. Inset of (b) shows the extended stability trace of nanowO $3\left|\mathrm{TiO}_{2}\right| \mathrm{NiO}_{x}$. The activity of the mesolTO|PSII electrode was recorded in a $\mathrm{pH} 6.5$ MES electrolyte solution and the nanowO $\left|\mathrm{TiO}_{2}\right| \mathrm{NiO}_{x}$ electrode was recorded in a $\mathrm{pH} 9.2$ $\mathrm{B}_{\mathrm{i}}$ buffer solution $(0.1 \mathrm{M})$. 
more efficient co-catalyst might accelerate the water oxidation kinetics and alleviate the surface recombination in the low bias region.

Conversely, nanoWO $\mathrm{W}_{3}\left|\mathrm{TiO}_{2}\right| \mathrm{NiO}_{x}$ has a clear advantage in terms of photocurrent density per geometrical surface area due to its higher catalyst density compared to mesoITO|PSII, which is important for applications. Although nano $\mathrm{WO}_{3}\left|\mathrm{TiO}_{2}\right| \mathrm{NiO}_{x}$ displays a higher photocurrent density than mesoITO|PSII, the photocurrent is still much lower than its theoretical photocurrent (approximately $5 \mathrm{~mA} \mathrm{~cm}{ }^{-2}$ ). ${ }^{21}$ To boost further the photocurrent plateau, charge recombination within the nano $\mathrm{WO}_{3}\left|\mathrm{TiO}_{2}\right| \mathrm{NiO}_{x}$ must be minimised. Combination with another semiconductor to form a $\mathrm{p}-\mathrm{n}$ junction,${ }^{22}$ increasing intrinsic conductivity by elemental doping, ${ }^{23}$ and suppression of electron back injection with an oxide underlayer $^{24}$ are possible strategies to enhance further the photocurrent of this synthetic electrode.

Inorganic semiconductors offer a more robust approach to provide the oxidising potentials necessary to photo-oxidise water than semi-biological systems. In this study, $\mathrm{WO}_{3}$ was interfaced with the $\mathrm{TiO}_{2}$ and $\mathrm{NiO}_{x}$ composite by a simple and scalable deposition of a molecular precursor to improve its photo-activity and stability. NanoWO $\mathrm{WO}_{3}\left|\mathrm{TiO}_{2}\right| \mathrm{NiO}_{x}$ is considerably more stable than mesoITO|PSII at photo-oxidative conditions (Fig. 5 and Table 1).

\section{Conclusions}

An enzymatic photoanode consisting of PSII immobilised on mesoITO and a synthetic photoanode consisting of a $\mathrm{TiO}_{2}$ and $\mathrm{NiO}_{x}$ composite on a $\mathrm{WO}_{3}$ semiconductor operational under near $\mathrm{pH}$-neutral conditions have been discussed. $\mathrm{NanoWO}_{3}\left|\mathrm{TiO}_{2}\right| \mathrm{NiO}_{x}$ mimics the key functionalities of PSII, namely, light absorption, charge separation and water oxidation catalysis. As expected, the highly evolved PSII displays a higher turnover frequency than $\mathrm{NiO}_{x}$ on nano $\mathrm{WO}_{3}\left|\mathrm{TiO}_{2}\right| \mathrm{NiO}_{x}$. Bulk and surface charge recombination is currently a major hurdle in achieving high photon-to-current conversion efficiencies. The resistance to charge recombination of PSII is evident from the lower applied potential required to reach its saturation photocurrent in the mesoITO|PSII system compared with nano $\mathrm{WO}_{3}\left|\mathrm{TiO}_{2}\right| \mathrm{NiO}_{x}$. Thus the enzymatic system exhibits less surface charge recombination events at a low overpotential region compared with the synthetic system. In this respect, mesoITO|PSII behaves more like an "ideal" photocatalyst. The IPCE spectra confirm that PSII has the ability to promote electron transfer with low energy photons $(680 \mathrm{~nm})$, whereas $\mathrm{WO}_{3}$ is only capable of utilising high-energy photons $(<465 \mathrm{~nm})$ to achieve water oxidation at a similar onset potential.

Nevertheless, the nanoWO $\mathrm{W}_{3}\left|\mathrm{TiO}_{2}\right| \mathrm{NiO}_{x}$ electrode displays higher IPCEs and a more than three orders of magnitude higher photocurrent density than mesoITO| PSII due to the high $\mathrm{NiO}_{x}$ catalyst density on the synthetic electrode compared to the low PSII loading. Another distinct advantage of the synthetic over the enzymatic photoanode is the greater photo-stability under solar light irradiation due to the lack of repair machinery in the isolated PSII. In summary, our synthetic system will show promise for applications in water splitting in the long-term if important lessons from PSII can be learnt: longer wavelength absorption will boost the overall solar energy conversion efficiency and saturation currents at a more negative potential decrease heat losses in an operating device. 


\section{Acknowledgements}

Financial support from EPSRC (EP/H00338X/2 to E. R. and nanoDTC to D. M.), the Christian Doppler Research Association (Austrian Federal Ministry of Science, Research and Economy and National Foundation for Research, Technology and Development), the OMV Group (to E. R.) and Cambridge Trust (to Y.-H. L.) are gratefully acknowledged. We thank Mr Timothy C. King and Prof. Dominic S. Wright for a kind gift of $\left[\mathrm{Ti}_{2}(\mathrm{OEt})_{9}(\mathrm{NiCl})\right]_{2}$. Dr Tanai Cardona and Prof. A. William Rutherford provided us with a sample of photosystem II. We also thank Dr Hyun S. Park, Miss Claire Wombwell and Prof. Rutherford for valuable comments on the manuscript.

\section{References}

1 (a) Y. Tachibana, L. Vayssieres and J. R. Durrant, Nat. Photonics, 2012, 6, 511; (b) S. Y. Reece, J. A. Hamel, K. Sung, T. D. Jarvi, A. J. Esswein, J. J. H. Pijpers and D. G. Nocera, Science, 2011, 334, 645; (c) C.-Y. Lin, Y.-H. Lai, D. Mersch and E. Reisner, Chem. Sci., 2012, 3, 3482; (d) L. Liao, Q. Zhang, Z. Su, Z. Zhao, Y. Wang, Y. Li, X. Lu, D. Wei, G. Feng, Q. Yu, X. Cai, J. Zhao, Z. Ren, H. Fang, F. Robles-Hernandez, S. Baldelli and J. Bao, Nat. Nanotechnol., 2014, 9, 69; (e) J. R. McKone, N. S. Lewis and H. B. Gray, Chem. Mater., 2014, 26, 407; $(f)$ W. D. Chemelewski, H.-C. Lee, J.-F. Lin, A. J. Bard and C. B. Mullins, J. Am. Chem. Soc., 2014, 136, 2843; (g) P. M. Rao, L. Cai, C. Liu, I. S. Cho, C. H. Lee, J. M. Weisse, P. Yang and X. Zheng, Nano Lett., 2014, 14, 1099; (h) B. M. Klepser and B. M. Bartlett, J. Am. Chem. Soc., 2014, 136, 1694; (i) C.-Y. Lin, D. Mersch, D. A. Jefferson and E. Reisner, Chem. Sci., 2014, 5, 4906. 2 M. Kato, J. Z. Zhang, N. Paul and E. Reisner, Chem. Soc. Rev., 2014, 43, 6485.

3 (a) K. N. Ferreira, T. M. Iverson, K. Maghlaoui, J. Barber and S. Iwata, Science, 2004, 303, 1831; (b) H. Dau and I. Zaharieva, Acc. Chem. Res., 2009, 42, 1861; (c) Y. Umena, K. Kawakami, J.-R. Shen and N. Kamiya, Nature, 2011, 473, 55; (d) T. Cardona, A. Sedoud, N. Cox and A. W. Rutherford, Biochim. Biophys. Acta, 2012, 1817, 26.

4 (a) Y.-H. Lai, T. C. King, D. S. Wright and E. Reisner, Chem.-Eur. J., 2013, 19, 12943; (b) Y.-H. Lai, C.-Y. Lin, Y. Lv, T. C. King, A Steiner, N. M. Muresan, L. Gan, D. S. Wright and E. Reisner, Chem. Commun., 2013, 49, 4331.

5 (a) S. D. Tilley, M. Cornuz, K. Sivula and M. Grätzel, Angew. Chem., Int. Ed., 2010, 49, 6405; (b) W. J. Youngblood, S.-H. A. Lee, Y. Kobayashi, E. A. Hernandez-Pagan, P. G. Hoertz, T. A. Moore, A. L. Moore, D. Gust and T. E. Mallouk, J. Am. Chem. Soc., 2009, 131, 926.

6 (a) Y. Jiang, F. Li, B. Zhang, X. Li, X. Wang, F. Huang and L. Sun, Angew. Chem., Int. Ed., 2013, 52, 3398; (b) J. D. Megiatto Jr, A. Antoniuk-Pablant, B. D. Sherman, G. Kodis, M. Gervaldo, T. A. Moore, A. L. Moore and D. Gust, Proc. Natl. Acad. Sci. U. S. A., 2012, 109, 15578; (c) M. M. Najafpour, T. Ehrenberg, M. Wiechen and P. Kurz, Angew. Chem., Int. Ed., 2010, 49, 2233; (d) M. Wiechen, I. Zaharieva, H. Dau and P. Kurz, Chem. Sci., 2012, 3, 2330.

7 (a) M. Kato, T. Cardona, A. W. Rutherford and E. Reisner, J. Am. Chem. Soc., 2012, 134, 8332; (b) M. Kato, T. Cardona, A. W. Rutherford and E. Reisner, J. Am. Chem. Soc., 2013, 135, 10610. 
8 M. Risch, K. Klingan, J. Heidkamp, D. Ehrenberg, P. Chernev, I. Zaharieva and H. Dau, Chem. Commun., 2011, 47, 11912.

9 P. G. Hoertz, Z. Chen, C. A. Kent and T. J. Meyer, Inorg. Chem., 2010, 49, 8179.

10 (a) A. Boussac, F. Rappaport, P. Carrier, J. M. Verbavatz, R. Gobin, D. Kirilovsky, A. W. Rutherford and M. Sugiura, J. Biol. Chem., 2004, 279, 22809; (b) M. Sugiura and Y. Inoue, Plant Cell Physiol., 1999, 40, 1219.

11 S. Eslava, M. McPartlin, R. I. Thomson, J. M. Rawson and D. S. Wright, Inorg. Chem., 2010, 49, 11532.

12 (a) A. J. Bard and L. R. Faulkner, Electrochemical Methods: Fundamentals and Applications, John Wiley \& Sons, Inc., NewYork, 2nd edn, 2001; (b) A. Kay, I. Cesar and M. Grätzel, J. Am. Chem. Soc., 2006, 128, 15714.

13 T. W. Kim and K.-S. Choi, Science, 2014, 343, 990.

14 T. Shibamoto, Y. Kato, M. Sugiura and T. Watanabe, Biochemistry, 2009, 48, 10682.

15 R. M. Navarro, M. Consuelo Alvarez-Galvan, J. A. V. de la Mano, S. M. AlZahrani and J. L. G. Fierro, Energy Environ. Sci., 2010, 3, 1865.

16 L. Zhang, E. Reisner and J. J. Baumberg, Energy Environ. Sci., 2014, 7, 1402.

17 G. Raszewski, W. Saenger and T. Renger, Biophys. J., 2005, 88, 986.

18 O. Yehezkeli, R. Tel-Vered, J. Wasserman, A. Trifonov, D. Michaeli, R. Nechushtai and I. Willner, Nat. Commun., 2012, 3, 742.

19 A. W. Rutherford, A. Osyczka and F. Rappaport, FEBS Lett., 2012, 586, 603.

20 J. A. Seabold and K.-S. Choi, Chem. Mater., 2011, 23, 1105.

21 Z. Chen, T. F. Jaramillo, T. G. Deutsch, A. Kleiman-Shwarsctein, A. J. Forman, N. Gaillard, R. Garland, K. Takanabe, C. Heske, M. Sunkara, E. W. McFarland, K. Domen, E. L. Miller, J. A. Turner and H. N. Dinh, J. Mater. Res., 2010, 25, 3.

22 A. Paracchino, V. Laporte, K. Sivula, M. Grätzel and E. Thimsen, Nat. Mater., 2011, 10, 456.

23 A. J. E. Rettie, H. C. Lee, L. G. Marshall, J.-F. Lin, C. Capan, J. Lindemuth, J. S. McCloy, J. Zhou, A. J. Bard and C. B. Mullins, J. Am. Chem. Soc., 2013, 135, 11389.

24 T. Hisatomi, H. Dotan, M. Stefik, K. Sivula, A. Rothschild, M. Grätzel and N. Mathews, Adv. Mater., 2012, 24, 2699. 